\title{
Artificial Bee Colony Optimization Based Despeckling Framework for Ultrasound Images
}

\author{
Pradeep K. Gupta ${ }^{1, *}$, Shyam Lal ${ }^{2}$ and Farooq Husain ${ }^{3}$ \\ ${ }^{1}$ Department of E\&C Engineering, PSIT, Kanpur-209305, Uttar Pradesh, India \\ ${ }^{2}$ Department of E\&C Engineering, ,NIT Karnataka, Surathkal, Mangaluru-575025, India \\ ${ }^{3}$ Department of E\&C Engineering, SSITM, Aligarh Uttar Pradesh, India
}

Received 26 January 2020; Accepted 20 October 2020

\begin{abstract}
This paper proposed an artificial bee colony optimization (ABC) algorithm based despeckling framework to overcome the effect of speckle noise present in real ultrasound images. A low pass filter and fast non-local mean filter along with Artificial Bee Colony (ABC) optimization algorithm are used for the quality enhancement of ultrasound images. The output results obtained for the real ultrasound images filtered with the proposed approach and the other most studied approaches discussed in the literature. The outperformance of the proposed method is verified by calculation of peak signal to noise ratio (PSNR), mean square error (MSE), mean absolute error (MAE), and structure similarity index (SSIM) quality measures. The proposed filtering approach is tested on eight real clinical ultrasound images of adrenal gland, appendicitis, bladder, pancreas, parathyroid gland, scrotal gland, thoracic wall, and uterus. The experimental results yield that the quantitative and qualitative results of the proposed framework are better than benchmark despeckling methods compared to real ultrasound images. Further, the proposed framework also preserves the fine details in real ultrasound images.
\end{abstract}

Keywords: Optimization algorithm, Artificial Bee colony, Despeckling, Ultrasound Image, filtering.

\section{Introduction}

Currently, for the diagnosis of patients, there are so many modalities are available in the medical domain. Out of different imaging technique, ultrasound images has been used by a medical practitioner, to find out the desired information from the images to diagnosis the patients, other advantages of ultrasound imaging are its portability, cost-effectiveness, and non-invasive nature of the procedure. The relevant information extraction from real ultrasound images is a very tiresome task due to the low visual quality of the images. So there is a requirement of a procedure that improves the visual quality of real clinical ultrasound images. Currently, Ultrasound imaging is used in ophthalmology, cardiology, orthopedics, and gynecology. The visual analysis and quantitative analysis of these images are performed to find out the different diseases in the patients. But both kinds of research are difficult task in ultrasound imaging due to different artifacts presents in it. The various artifacts in ultrasound are occurred due to additive and multiplicative noise. The multiplicative noise component strongly affects the visual parameter of the image [1]. The visual qualities of Ultrasound images are governed by a unique noise know as speckle. Hence, there is a requirement of a framework to reduce it; researchers have been proposed a different type of speckle removing filters in the last two decades. Based on design approaches, the main filters are spatial filter and frequency domain filter. Speckle-noise reduction is

*E-mail address: pradeepsingla7@gmail.com ISSN: 1791-2377 @ 2020 School of Science, IHU. All rights reserved. doi:10.25103/jestr.135.04 performed carefully because it may contain important medical information of ultrasound imaging also [2].

The most studied spatial filter is the linear wiener filter and the mean filter [3-4]. The process of these filters are causing blurring on the edges of the images so that the visual appearance of the image is not good. In the early stage of development, the filtering approach was inspired by the approach that is used for SAR images, because of similar noise patterns present in these images[5]. Adaptive filters were also used to overcome the effect of this type of noise. Several different adaptive filters were also developed by different researchers [6-9] in previous years. These filters are also known as classical filters in literature. These filters model the speckle noise as a multiplicative signal model, in which the noise to be assumed as Gaussian distributed. The main disadvantage of classical filters is that they tend to remove the information of the tissue along with noise due to inappropriate speckle-noise modeling. To overcome the problem of classical filter, more elaborated filters are trying to define the edges of images by non-homogeneous diffusive phenomena of heat. The most discussed filters of this category are also model the speckle noise as a multiplicative noise model [1013]. In this filtering approach, the speckle pattern is erased during the diffusion of the image, which resulted in piecewise images. Although these filters perform better in comparison to early developed classical speckle removal filters are still suffered from the elimination of texture details of an image. After that, a new paradigm of speckle-noise removal filters is proposed; these filters used the concept of local filtering [14]. In this approach, the author used Pearson distance as the required criteria for patch comparison. The results show that this method is beneficial for smoothing homogeneous areas while preserving the edges of the images. 
Recently many authors have been applied swarm intelligence to design the filters for image denoising [15-17]. Boudjelaba et al. in [18] compared all evolutionary techniques for the designing of FIR filter. In this research, the author demonstrated that the filter coefficients could be optimized with the help of an evolutionary algorithm present in the literature. Latifoglu in [19] used an artificial bee colony algorithm to design a despeckling filter for the medical images. This approach differs in no of ways because it does not use the wavelet or Fourier domain. The main advantage of this system is that it is straightforward to implement for speckle noise elimination. Out of different evolutionary algorithm, the $\mathrm{ABC}$ is a nature-inspired algorithm which has many advantages in comparison to other nature-inspired algorithms. The main benefits of this algorithm are, it can be easily implemented, quick local convergence, and global optimization ability. To solve the problem, it uses the foraging behavior of honey bees [20].

The significant contributions of this research work are:

- A new filtering approach provides the enhanced output images for the clinical ultrasound images.

- The proposed filter contributes a significant role in the filtering of the speckle-noise present in real ultrasound images.

- Simulation experiments have been conducted over several real ultrasound images.

The remaining part of this work is as follows; preliminaries for the proposed are explained in section 2, section 3 illustrated the proposed optimization-based framework, section 4 Components of the proposed framework. The comparative performance analysis of the proposed filter with different filtering approaches is presented details in section 5 in the form of a result, and the last conclusion is present in section 6 .

\section{Preliminaries for Proposed framework}

\subsection{Noise Model}

The nature of the noise presents in these images is random nature. the noise is locally correlated and the effect of it multiplicative, defined in Eq. 1

$$
I_{n}(r, s)=I_{n f}(r, s) \times \sigma_{n}(r, s)
$$

where $I_{n}(r, s)$ represents the noisy image, $I_{n f}(r, s)$ is the noise-free image, and $\sigma_{n}(r, s)$ is the intensity of the noise.

\subsection{D Low Pass Filter}

The input reference image $I_{r}\left(r_{1}, r_{2}\right)$ for $2 \mathrm{D}$ FIR filter is generated by passing $P_{n}\left(r_{1}, r_{2}\right)$ through the $2 \mathrm{D}$ low pass filter. In this proposed framework same filter coefficients are used, which was already recommended in most of the literature and define as in Eq.2 and Eq.3

$$
\begin{aligned}
& H\left(z_{1}\right)=1-0.7 z_{1}^{-1}+0.5 z_{1}^{-2}-0.05 z_{1}^{-3}+0.0056 z_{1}^{-4}-0.0004 z_{1}^{-5} \\
& H\left(z_{2}\right)=1-0.7 z_{2}^{-1}+0.5 z_{2}^{-2}-0.045 z_{2}^{-3}+0.0046 z_{2}^{-4}-0.0003 z_{2}^{-5}
\end{aligned}
$$

\subsection{D-FIR Digital filter}

Generally, the denoising of the images is performed by the help of filters. The main filters used for denoising are FIR filter and IIR filter. Out of these two filter category, the IIR filter has a stability problem, so we prefer the FIR filter for our approach. Due to the high sensitivity of the human eye, the phase response of the system has the main concern; therefore, 2D FIR filters have a linear phase. Although the physical realization of FIR filter by software as well as in hardware is straightforward and it is always stable. The output of its defined as Eq.4.

$$
\begin{aligned}
& R\left(r_{1}, r_{2}\right)=\sum_{a=0}^{A-1} \sum_{b=0}^{B-1} Q(a, b) P_{n}\left(r_{1}, r_{2}\right) \\
& R\left(r_{1}, r_{2}\right) \text { is the output corresponding to noisy input }
\end{aligned}
$$

signal $P_{n}\left(r_{1}, r_{2}\right)$ and $Q(a, b)$ represents the weight matrix of $A \times B$ order filter. The output, input, and weight matrix of the FIR digital filter can be represented as:

$$
R\left(r_{1}, r_{2}\right)=\left[\begin{array}{ccc}
R\left(r_{1}, r_{2}\right) & \ldots & R\left(r_{1}, r_{2}-B+1\right) \\
\ldots & \ldots & \ldots \\
R\left(r_{1}-B+1, r_{2}\right) & \ldots & R\left(r_{1}-A+1, r_{2}-B+1\right)
\end{array}\right]
$$

$$
Q(a, b)=\left[\begin{array}{ccc}
Q(0,0) & \ldots & Q(0, B-1) \\
\ldots & \ldots & \ldots \\
Q(A-1,0) & \ldots & Q(A-1, B-1)
\end{array}\right]
$$

$$
P_{n}\left(r_{1}, r_{2}\right)=\left[\begin{array}{ccc}
P_{n}\left(r_{1}, r_{2}\right) & \ldots & P_{n}\left(r_{1}, r_{2}-B+1\right) \\
\ldots & \ldots & \ldots \\
P_{n}\left(r_{1}-A+1, r_{2}\right) & \ldots & P_{n}\left(r_{1}-A+1, r_{2}-B+1\right)
\end{array}\right]
$$

2D digital FIR filter maintained the conditions of BIBO i.e. bounded input produces bounded output; hence the filter is always stable.

\subsection{Fast Non-Local Mean (FNLM) Filter}

The proposed filtering approach uses Non-local mean filtering to improve the results. The principle of FNLM filter is also based on non-local filtering [21]. In the patch-based approach, the local comparison of the image pixel is performed by the nonlocal comparison of patches. Non-Local filtering has no assumption for the most relevant image pixel to denoise the current pixel. Hence it works on available information redundancy in the image. Non-local filtering analyzes the pixel pattern in the image rather than intensity. NL-means filter works on two different approaches:

1) Pixel-wise Approach: in this approach The resorted intensity of the pixel $p_{i}$ for a noisy image $P_{n}=\left(P_{n}\left(p_{i}\right)\right)_{p_{i} \in \Omega^{d}}$ which is defined over a bounded region $\Omega^{d} \subset \Re^{d}$ (rectangle of size $\left.\left|\Omega^{d}\right|\right)$ and $P_{n}\left(p_{i}\right) \in \Re_{+}$is calculated as : 
$N L\left(P_{n}\right)\left(p_{i}\right)=\sum_{p_{j} \in \Omega^{d}} Q\left(p_{i}, p_{j}\right) P_{n}\left(p_{j}\right)$

$w\left(p_{i}, p_{j}\right)$ is represent the weight used for restoring the

value $p_{n}\left(p_{i}\right)$ given $P_{n}\left(p_{j}\right)$ and based on the similarity between

$P_{n}\left(N_{i}\right)$ and $P_{n}\left(N_{j}\right)$

2) Block-wise approach: the block-wise approach reduces the complexity of the pixel-wise approach of NL-means filtering. This approach consists of three steps;

i) The total volume is divided into blocks with overlapping allow i.e.

$\Omega^{d}=\cup_{b} B_{i b}$

where $B_{i b}$ represents overlapping blocks.

ii) Applying pixel-wise operation on each block, restoring the block $B_{i b}$ as follows:

$$
N L\left(P_{n}\right)\left(B_{i b}\right)=\sum_{B_{j} \in S_{j}^{d}} Q\left(B_{i b}, B_{j}\right) P_{n}\left(B_{j}\right)
$$

iii) Replacing the pixel intensities from the restored blocks by calculating the mean of the restored values $N L\left(P_{n}\left(B_{i b}\right)\right)$.

In fast nonlocal mean filter, we used the blockwise approach along with PCA to calculate the different coefficient so that the speed of the procedure should be increase.

\section{Proposed Framework}

In this framework, the weights of despeckling filters are calculated by $\mathrm{ABC}$ algorithm. Recently Karaboga and Basturk [20] proposed an optimization algorithm that procedure analogue the behavior of honey bees, known as artificial bee colony $(\mathrm{ABC})$ algorithm. It provides an efficient solution to complex problems of different research fields. In this algorithm finding the optimum solution of a problem is directly associated with the way of searching the best food source by the honeybees and it is optimized according to the fitness evaluations criteria. The origin of food refers to solution space, while the size of the solution is the value of the parameter for an expected solution. All the bees that help in the searching work are known as employed bees, onlookers, and scout bees, respectively. The number of solutions, employed bees, and onlooker bees are equal. The employed bees exploit all possibilities of food sources and convey all extracted information to the onlooker bees for the next step. Based on information received, the Onlooker bees decide which food source is exploited next. If employed bees are not able to search food sources, then they convert to scout bees. Hence the duty of scout bees is to find new food sources randomly.

The different initialization parameters for this algorithm are:
(1). The initial population is equal to the employee or onlooker bees.

(2). Employed bees convert into scout bees when all food sources are exploited.

(3). The Maximum No. of iteration of the process is limited by the number of food sources.

In this proposed framework $\mathrm{ABC}$ optimization algorithm is used in pre-filtering along with fast non-local filtering. The schematic of the proposed filtering framework is shown in Fig.1

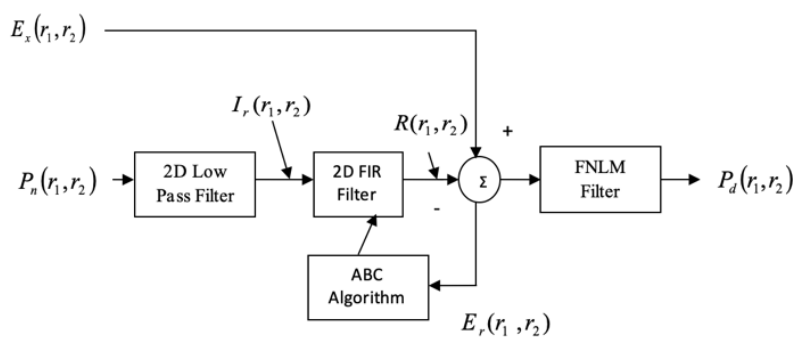

Fig.1. Block Diagram of Proposed Filtering Approach

The $\mathrm{ABC}$ algorithm optimizes the filter coefficient as per the objective function of the proposed framework. The weight matrix corresponding to the coefficient matrix and the input image matrix is represented as their 1D lexicographic form [22] described as:

$$
\begin{gathered}
Q_{i}(a, b)=[Q(0,0) \ldots Q(0, B-1) \ldots Q(1,0) \ldots \times Q(A-1, B-1)]^{T} \\
P_{n}\left(r_{1}, r_{2}\right)=\left[\begin{array}{l}
P_{n}\left(r_{1}, r_{2}\right) \ldots P_{n}\left(r_{1}, r_{2}-B+1\right) \ldots P_{n}\left(r_{1}-1, r_{2}\right) \\
\ldots \times P_{n}\left(r_{1}-A+1, r_{2}-B+1\right)
\end{array}\right]^{T}
\end{gathered}
$$

where $i$ represent the iteration number, and the corresponding output signal can be represented as

$$
R\left(r_{1}, r_{2}\right)=Q_{i}^{T}\left(r_{1}, r_{2}\right) P_{n}\left(r_{1}, r_{2}\right)
$$

the error signal $E_{r}\left(r_{1}, r_{2}\right)$ is generated by the difference between the expected signal $E_{x}\left(r_{1}, r_{2}\right)$ and the actual output $R\left(r_{1}, r_{2}\right)$ of FIR filter. So we can define the error signal as:

$$
E_{r}\left(r_{1}, r_{2}\right)=E_{x}\left(r_{1}, r_{2}\right)-R\left(r_{1}, r_{2}\right)
$$

In order to adaptively adjust the filter coefficients, the error signal is feedback in the proposed approach, and hence the coefficient of the filter is adaptively adjusted. The objective function for the proposed algorithm is to minimize the MSE between the expected signal $E_{x}\left(r_{1}, r_{2}\right)$ and the actual output $R\left(r_{1}, r_{2}\right)$ of the FIR filter; hence by minimizing the objective function, we obtained the optimum weight matrix.

$O(Q(a, b))=\frac{1}{R_{1} R_{2}} \sum_{r_{1}=0}^{R_{1}-1} \sum_{m_{r_{2}}=0}^{R_{2}-1}\left[E_{x}\left(r_{1}, r_{2}\right)-R\left(r_{1}, r_{2}\right)\right]^{2}$ 
By using Eq.15, the proposed filtering approach optimizes the coefficients of the weight matrix as per the requirement of the objective function.

The different steps used in the proposed framework are systematically shown in flow process of Fig. 2

Step 1: Represent the weight matrix and input image signal as a lexicographic form.

Step 2: Fix the possible number of solutions present in solution space $\left(f_{i j}\right): f_{i j}, i=1, \ldots \ldots . S_{N}, j=1, \ldots \ldots . . C_{N} \quad\left(S_{N}\right.$ : number of solution) ( $C_{N}$ : number of coefficient in the filter) Step 3: Calculate the fitness value for the given solution by using equation (15)

Step 4: Iteration $i_{n}=1$

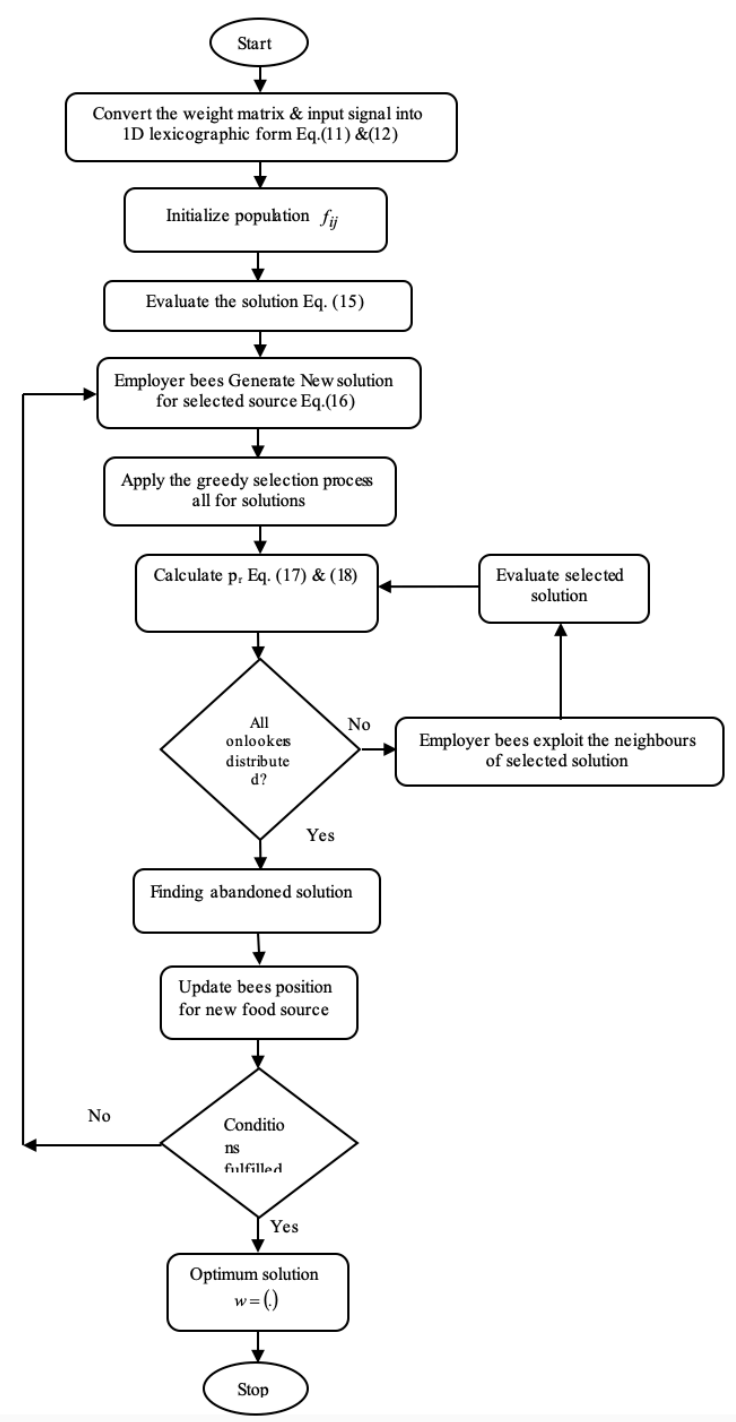

Fig. 2. Process Flow of Filter Coefficients Optimization by ABC Algorithm

Step 5: Generate new solutions for the FIR filter $\left(f_{i j}^{\text {new }}\right)$ by using the equation

$$
f_{i j}^{\text {new }}=f_{i j}+\beta_{i j}\left(f_{i j}-f_{k j}\right)
$$

for employed bees where $j \in\left(1,2 \ldots . . C_{N}\right)$ and $k \in\left(1,2 \ldots . S_{N}\right)$ is a randomly chosen index and $\beta_{i j} \in[-1,1]$

Step 6: Select the best solutions from all $\left(f_{i j}^{n e w}\right)$ generated by employed bees and $\left(f_{i j}\right)$ by the help of a greedy selection procedure.

Step 7: Calculate the probability value $p_{r}$ for the $r^{\text {th }}$ solution $f_{r}$ using equations (15) and (16)

$$
\begin{aligned}
& \text { fit }_{r}(Q(a, b))=\frac{1}{1+O_{r}(Q(a, b))} \\
& p_{r}=\frac{f i t_{r}}{\sum_{i=1}^{S_{N}} f i t_{i}}
\end{aligned}
$$

Where $\operatorname{fit}_{r}(Q(a, b))$ is the fitness value for the $r^{\text {th }}$ solution and $O_{r}$ is the value of the objective function for the $r^{\text {th }}$ solution.

Step 8: Generate a new solution for the onlooker bees based on $f_{r}$ and $p_{r}$ analyze them by using Eq.15

Step 9: Select the best solutions from all $\left(f_{i j}^{\text {new }}\right)$ generated by onlooker bees and $\left(f_{i j}\right)$ with the help of a greedy selection procedure.

Step 10: find out the abandoned solution for the scout bees if exists and replace $f_{r}$ by $f_{r}^{\text {new }}$.

Step11: Store the best solution till now.

Step 12: Increment the iteration by $i_{n}=i_{n}+1$

Until $i_{n}=i_{n}^{\max }(\operatorname{maximum}$ iteration number)

\section{Components of Proposed Framework:}

The robustness of the proposed filtering approach is observed by comparing the proposed framework T-10: with the existing filtering methods : T-1: SRAD filter [10], T-2: DPAD filter [11], T-3: Wiener filter [4], T-4: Least Mean Square filter [23], T-5: Non Local Means filter [21], T-6: OBNLM filter [14], T-7: Anisotropic Diffusion filter [25], in spite of these filters, we compare our proposed work with the recently proposed algorithm such as T-8: filtering method based on ABC algorithm [19], and T-9: 2D-ABC adaptive filtering algorithm proposed in [26].

\subsection{Open source database}

For the purpose of quantitative and qualitative comparison of different filtering approaches with our proposed work, a simulation experiment is conducted on the real ultrasound images of adrenal gland, appendicitis, bladder, pancreas, parathyroid gland, scrotal gland, thoracic wall, and uterus. These images are available on an open-source database (http:// www.ultrasoundcases.info).

\subsection{Quality measures}


In image despeckling methods, the error is measured with the help of mean absolute error (MAE) and mean square error (MSE) defined as respectively

$$
\begin{aligned}
& M A E=\frac{1}{A B} \sum_{r_{1}=0}^{A-1} \sum_{r_{2}=0}^{B-1}\left\|P_{d}\left(r_{1}, r_{2}\right)-P_{n}\left(r_{1}, r_{2}\right)\right\| \\
& M S E=\frac{1}{A B} \sum_{r_{1}=0}^{A-1} \sum_{r_{2}=0}^{B-1}\left(P_{d}\left(r_{1}, r_{2}\right)-P_{n}\left(r_{1}, r_{2}\right)\right)^{2}
\end{aligned}
$$

$P_{d}\left(r_{1}, r_{2}\right)$ and $P_{n}\left(r_{1}, r_{2}\right)$ are the output and input value for an image of order $A \times B$.

The quality of the enhancement is measured by calculating the value of peak signal to noise ratio (PSNR) defined as

$$
\operatorname{PSNR}(d B)=10 \log _{10}\left(\frac{I_{M A X}^{2}}{M S E}\right)
$$

Where $I_{M A X}$ is the maximum power of the input signal. High PSNR and Low MSE value are desirable for a good method.

The similarity between input and output is measured by the structural similarity index (SSIM) defined as [27].

$$
\operatorname{SSIM}(I, O)=\frac{\left(2 \mu_{I} \mu_{O}+c_{1}\right)\left(2 \sigma_{I, O}+c_{2}\right)}{\left(\mu_{I}^{2}+\mu_{O}^{2}+c_{1}\right)\left(\sigma_{I}^{2}+\sigma_{O}^{2}+c_{2}\right)}
$$

$I$ and $O$ are the input and output signals.
$\mu_{I}, \sigma_{I}^{2}, \mu_{O}, \sigma_{O}^{2}$ and $\sigma_{I, O}$ are mean, variance, covariance values for noisy and denoise images, respectively. $\left(c_{1}, c_{2}\right)$ are constants for equation stabilization factors.

\section{Results and Discussion}

The performance analysis of the proposed framework is performed by conducting a simulation experiment on real ultrasound images. In the experiment, the noisy images were obtained by adding different noise intensity in the real ultrasound images. We calculate the different quality measures and get output image for all the despeckling methods. The visual results and numerical values of quality metrics of the proposed framework are compared with the nine most prominent benchmark despeckling filters. We calculate the quality measures for nine benchmark despeckling filters on the eight different ultrasound images and compared these results with the proposed filtering framework. To optimize the filter coefficient in the proposed framework, ABC optimization was used due to its simplicity. The value of the controlling parameter of the proposed algorithm $S_{N}, C_{N}$ and $i_{t}^{\max }$ is 50,9 , and 100 , respectively. The controlling parameters of benchmark despeckling filters were taken, as suggested by the respective authors in the literature.

To justify the robustness of the proposed filter, we find out the results for eight different ultrasound images of adrenal gland, appendicitis, bladder, pancreas, parathyroid gland, scrotal gland, thoracic wall, and uterus. During the experiment, the noisy images are obtained by adding the noise variance $\sigma=0.1,0.2$ in the original image. The numerical values of PSNR, MSE, MAE, SSIM for different despeckling filters are listed in Tables 1-8, and the visual result for different real clinical ultrasound images for different despeckling filters are shown in Fig.3 -18.

Fig.3 to Fig.18 shows the comparative visual performance of the proposed filtering framework with the nine prominent despeckling methods on the images of adrenal gland, appendicitis, bladder, pancreas, parathyroid gland, scrotal gland, thoracic wall, and uterus respectively at different noise variance levels $\sigma=0.1,0.2$. The comparative results of the proposed

$\underline{\text { Table 1. Comparative Quality Measures values for adrenal gland image }}$

\begin{tabular}{c|c|c|c|c|c|c|c|c}
\hline & \multicolumn{9}{c}{$\boldsymbol{\sigma}=\mathbf{0 . 1 0}$} & \multicolumn{4}{c}{$\boldsymbol{\sigma}=\mathbf{0 . 2 0}$} \\
\hline Filter & PSNR & MSE & MAE & SSIM & PSNR & MSE & MAE & SSIM \\
\hline T-1 & 22.22007 & 390.00801 & 10.76636 & 0.63495 & 22.12321 & 398.80366 & 10.44870 & 0.66082 \\
T-2 & 9.14023 & & 74.27299 & 0.03388 & 9.13986 & & 74.27297 & 0.03389 \\
& & 7926.06191 & & & dB & 7926.73034 & & \\
T-3 & 30.94221 & 52.34313 & 5.08799 & 0.87207 & 27.59813 & 113.04924 & 6.96563 & 0.81254 \\
T-4 & 26.26056 & 153.82396 & 6.68511 & 0.81609 & 20.40258 & 592.68326 & 14.05532 & 0.55877 \\
T-5 & 29.58977 & 71.46658 & 6.36767 & 0.73523 & 28.12013 & 100.24613 & 7.14563 & 0.72906 \\
T-6 & 29.82339 & 67.72386 & 6.06075 & 0.75615 & 26.23280 & 154.81058 & 8.81003 & 0.74236 \\
T-7 & 22.89403 & 333.94701 & 10.63129 & 0.66582 & 21.46161 & 464.42948 & 12.19507 & 0.61234 \\
T-8 & 18.35573 & 949.52771 & 8.78977 & 0.86019 & 18.48883 & 920.86828 & 10.10975 & 0.80218 \\
T-9 & 25.62818 & 177.93559 & 8.98163 & 0.78354 & 23.68959 & 278.04914 & 11.49598 & 0.66516 \\
T-10 & $\mathbf{3 1 . 2 6 4 7 9}$ & $\mathbf{4 8 . 5 9 6 0 8}$ & $\mathbf{4 . 8 9 9 1 0}$ & $\mathbf{0 . 9 0 5 5 8}$ & $\mathbf{2 8 . 6 0 0 0 8}$ & $\mathbf{8 9 . 7 5 7 7 5}$ & $\mathbf{6 . 7 6 9 6 0}$ & $\mathbf{0 . 7 5 9 7 6}$ \\
\hline
\end{tabular}

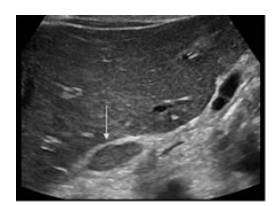

Real Image

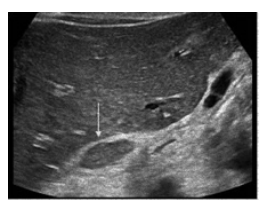

Noisy Image

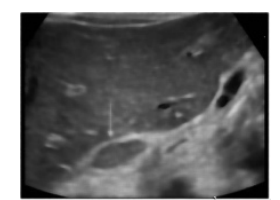

$\mathrm{T}-1$

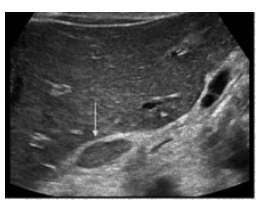

$\mathrm{T}-2$

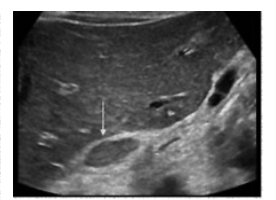

$\mathrm{T}-3$

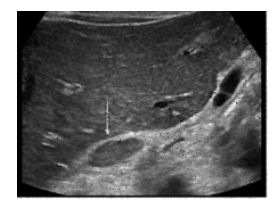

$\mathrm{T}-4$ 


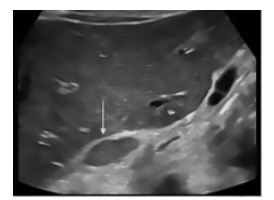

T-5

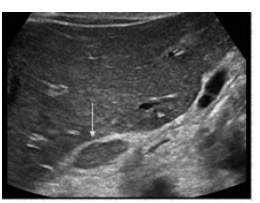

T-6

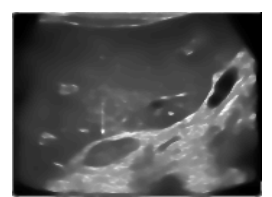

T-7

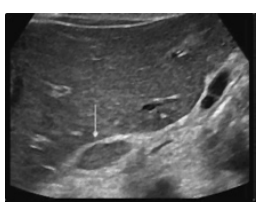

T-8

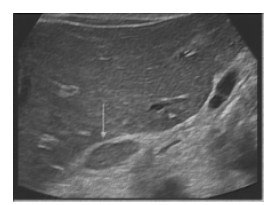

T-9

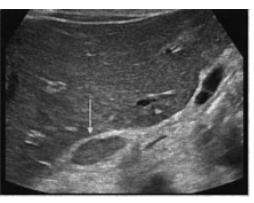

T-10

Fig. 3 Comparative visual performance of adrenal gland image at noise variance $\sigma=0.10$

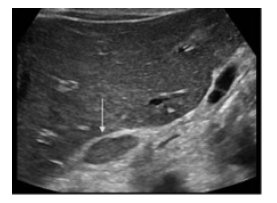

Real Image

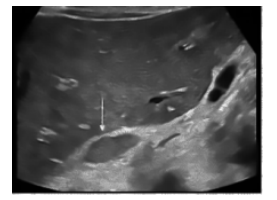

T-5

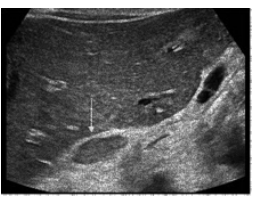

Noisy Image

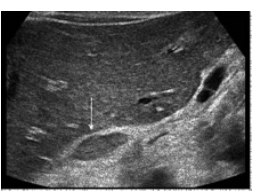

T-6

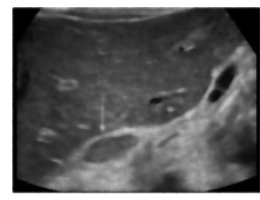

T-1

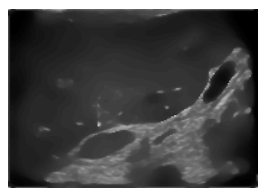

T-7

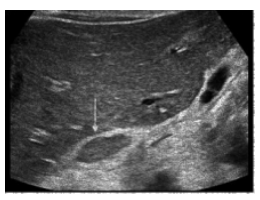

$\mathrm{T}-2$

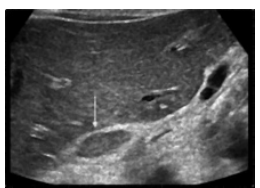

T-8

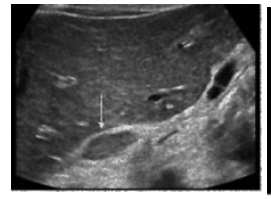

T-3

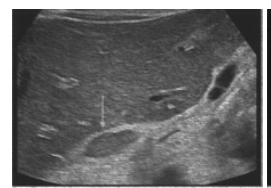

T-9

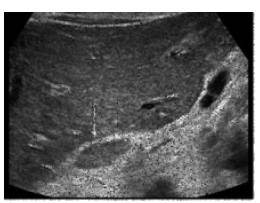

T-4

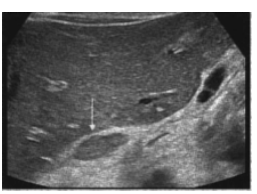

$\mathrm{T}-10$

Fig. 4. Comparative visual performance of adrenal gland image at noise variance $\sigma=0.20$

Table 2. Comparative Quality Measures values for appendicitis image

\begin{tabular}{c|c|c|c|c|c|c|c|c}
\hline & \multicolumn{4}{|c}{$\boldsymbol{\sigma}=\mathbf{0 . 1 0}$} & \multicolumn{4}{c}{$\boldsymbol{\sigma}=\mathbf{0 . 2 0}$} \\
\hline Filter & PSNR & MSE & MAE & SSIM & PSNR & MSE & MAE & SSIM \\
\hline T-1 & 22.28476 & 384.24114 & 10.13732 & 0.74913 & 22.42340 & 372.16914 & 10.31160 & 0.72951 \\
T-2 & 10.80170 & 5406.42122 & 57.92872 & 0.04798 & 10.80099 & 5407.30757 & 57.92867 & 0.04801 \\
T-3 & 31.02511 & 51.35338 & 4.75764 & 0.90671 & 28.37216 & 94.59415 & 6.03267 & 0.87062 \\
T-4 & 27.88228 & 105.88936 & 5.18370 & 0.87408 & 22.50491 & 365.24935 & 10.73385 & 0.67295 \\
T-5 & 31.46478 & 46.40907 & 5.10364 & 0.82662 & 27.33261 & 120.17641 & 7.61761 & 0.80295 \\
T-6 & 29.89631 & 66.59625 & 5.98844 & 0.79259 & 29.01734 & 81.53547 & 6.42978 & 0.78825 \\
T-7 & 22.28550 & 384.17623 & 6.48729 & 0.91535 & 22.23751 & 388.44518 & 8.52422 & 0.85067 \\
T-8 & 18.88895 & 839.81884 & 8.10294 & 0.90473 & 18.77784 & 861.58173 & 8.89549 & 0.86481 \\
T-9 & 23.83444 & 268.92818 & 9.68002 & 0.87048 & 22.95041 & 329.63930 & 11.65377 & 0.67368 \\
\hline T-10 & $\mathbf{3 1 . 8 8 7 9 9}$ & $\mathbf{4 2 . 0 9 9 9 6}$ & $\mathbf{4 . 4 9 2 0 6}$ & $\mathbf{0 . 9 2 1 0 9}$ & $\mathbf{2 9 . 6 5 4 4 1}$ & $\mathbf{7 0 . 4 1 0 7 7}$ & $\mathbf{5 . 8 5 6 2 5}$ & $\mathbf{0 . 8 1 8 6 0}$ \\
\hline
\end{tabular}

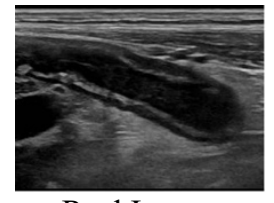

Real Image

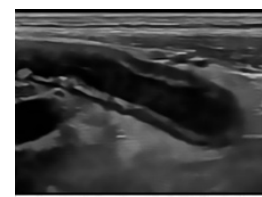

T-5

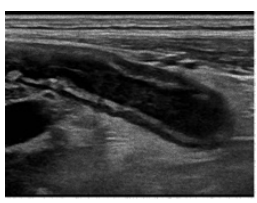

Noisy Image

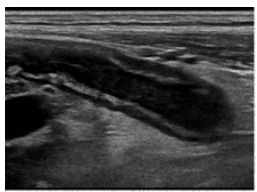

T-6

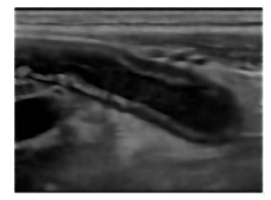

T-1

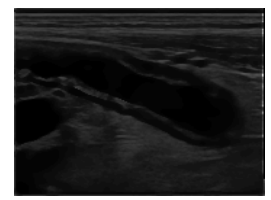

T-7

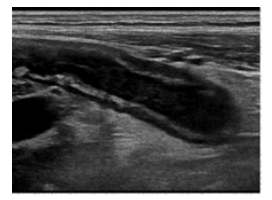

T-2

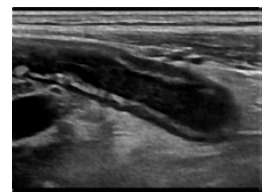

T-8

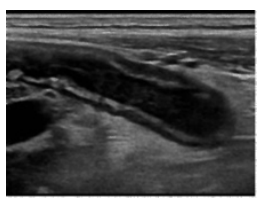

T-3

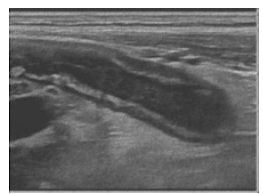

T-9

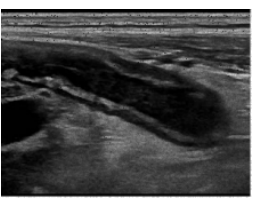

T-4

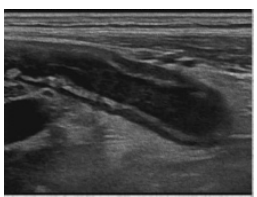

$\mathrm{T}-10$

Fig. 5. Comparative visual performance of appendicitis image at noise variance for the value of $\sigma=0.10$

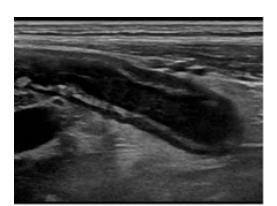

Real Image

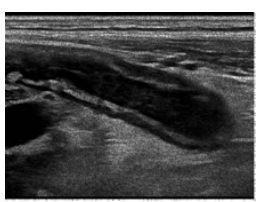

Noisy Image

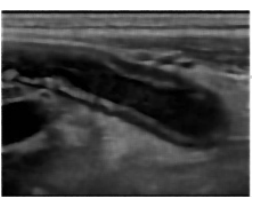

T-1

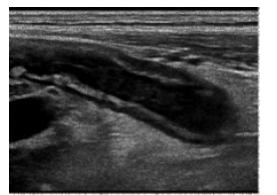

T-2

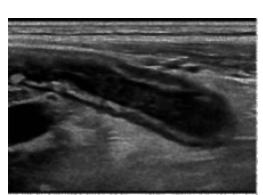

T-3

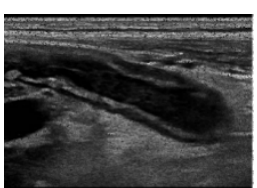

T-4 


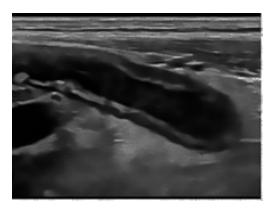

T-5

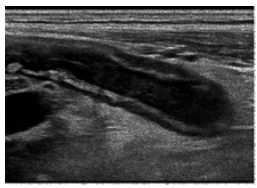

T-6

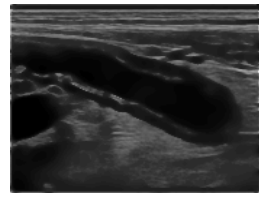

T-7

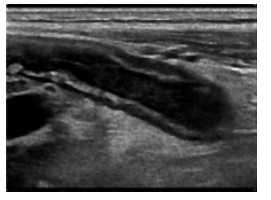

T-8

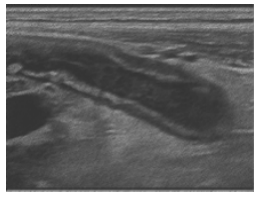

T-9

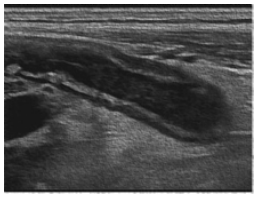

$\mathrm{T}-10$

Fig. 6. Comparative visual performance of appendicitis image at noise variance for the value of $\sigma=0.20$

Table 3. Comparative Quality Measures values for bladder image

\begin{tabular}{c|c|c|c|c|c|c|c|c}
\hline & \multicolumn{4}{|c|}{$\boldsymbol{\sigma}=\mathbf{0 . 1 0}$} & \multicolumn{4}{c}{$\boldsymbol{\sigma}=\mathbf{0 . 2 0}$} \\
\hline Filter & PSNR & MSE & MAE & SSIM & PSNR & MSE & MAE & SSIM \\
\hline T-1 & 24.28010 & 242.70018 & 6.01212 & 0.82268 & 24.09013 & 253.55228 & 5.63566 & 0.85456 \\
T-2 & 10.32218 & 6037.57254 & 51.92761 & 0.25781 & 10.32141 & 6038.63696 & 51.92759 & 0.25786 \\
T-3 & 34.71692 & 21.94769 & 2.56721 & 0.93831 & 28.92330 & 83.32027 & 4.50673 & 0.86782 \\
T-4 & 28.91946 & 83.39388 & 4.45341 & 0.85225 & 21.83512 & 426.15581 & 9.70799 & 0.67946 \\
T-5 & 30.89542 & 52.91004 & 4.46089 & 0.81394 & 27.13812 & 125.68071 & 6.59742 & 0.76794 \\
T-6 & 29.69893 & 69.69273 & 4.95591 & 0.79835 & 28.89018 & 83.95807 & 5.20200 & 0.79759 \\
T-7 & 23.16530 & 313.72576 & 7.32167 & 0.84219 & 22.45928 & 69.10688 & 8.36799 & 0.80090 \\
T-8 & 19.74489 & 689.59000 & 5.95651 & 0.92740 & 19.67348 & 701.02297 & 7.76771 & 0.87403 \\
T-9 & 23.95643 & 261.47951 & 8.76211 & 0.75017 & 24.08535 & 253.83121 & 9.12148 & 0.69856 \\
\hline T-10 & $\mathbf{3 0 . 9 3 9 8 9}$ & $\mathbf{5 2 . 3 7 1 0 6}$ & $\mathbf{4 . 3 0 7 8 1}$ & $\mathbf{0 . 8 7 1 6 0}$ & $\mathbf{2 9 . 9 4 9 1 0}$ & $\mathbf{6 5 . 7 9 1 6 6}$ & $\mathbf{4 . 7 4 3 6 3}$ & $\mathbf{0 . 8 1 3 9 2}$ \\
\hline
\end{tabular}

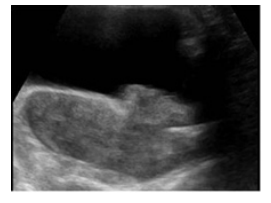

Real Image

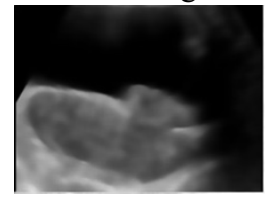

T-5

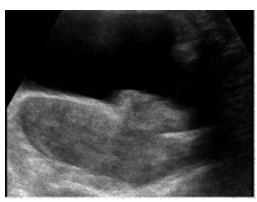

Noisy Image

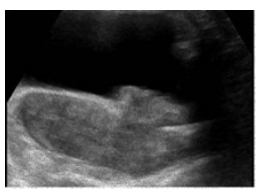

T-6

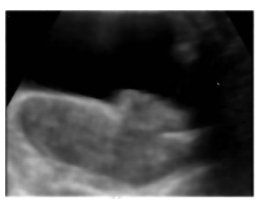

T-1

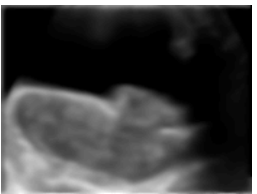

T-7

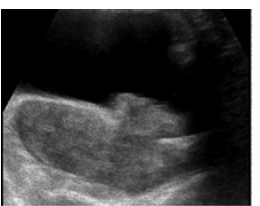

$\mathrm{T}-2$

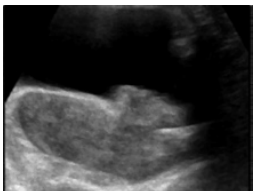

T-8

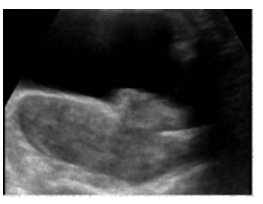

T-3

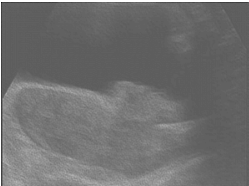

T-9

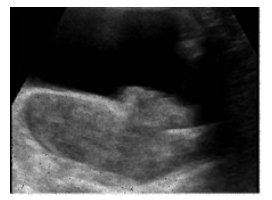

T-4

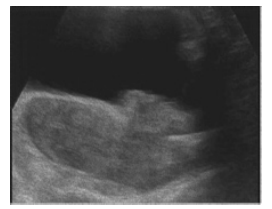

T-10

Fig. 7. Comparative visual performance of bladder image at noise variance for the value of $\sigma=0.10$

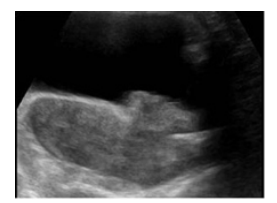

Real Image

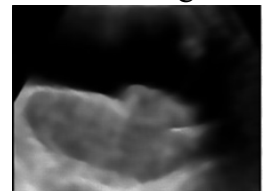

T-5

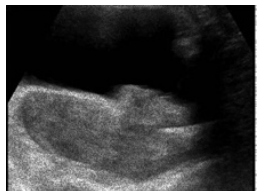

Noisy Image

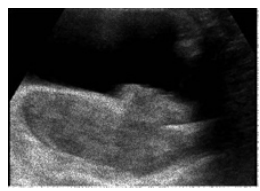

T-6

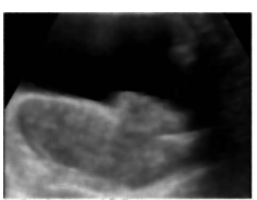

T-1

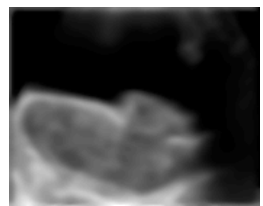

T-7

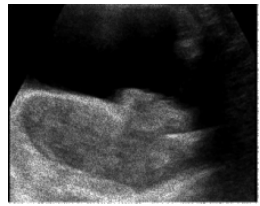

T-2

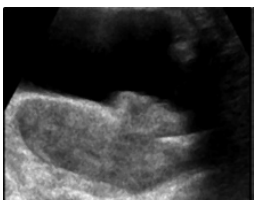

T-8

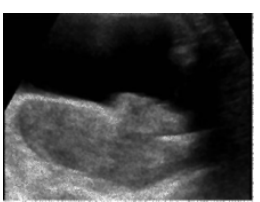

T-3

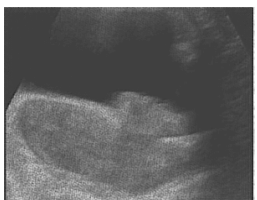

T-9

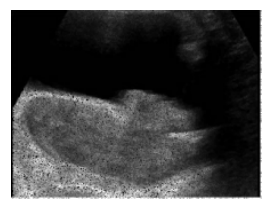

T-4

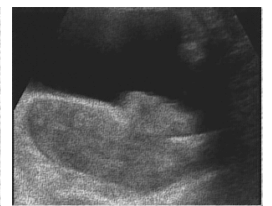

T-10

Fig. 8. Comparative visual performance of bladder image at noise variance for the value of $\sigma=0.20$

Table 4. Comparative Quality Measures values for pancreas image

\begin{tabular}{c|c|c|c|c|c|c|c|c}
\hline \multicolumn{1}{|c|}{} & $\boldsymbol{\sigma}=\mathbf{0 . 1 0}$ & $\boldsymbol{\sigma}=\mathbf{0 . 2 0}$ \\
\cline { 1 - 7 } Filter & PSNR & MSE & MAE & SSIM & PSNR & MSE & MAE & SSIM \\
\hline T-1 & 22.87196 & 335.64858 & 8.76861 & 0.74437 & 22.69103 & 349.92701 & 8.54327 & 0.76733 \\
T-2 & 10.52574 & 5761.11424 & 59.51635 & 0.08844 & 10.52518 & 5761.86041 & 59.51630 & 0.08847 \\
T-3 & 31.51453 & 45.88045 & 4.09551 & 0.92196 & $\mathbf{2 8 . 1 4 1 8 0}$ & $\mathbf{9 9 . 7 4 7 1 8}$ & $\mathbf{6 . 7 5 7 7 7}$ & $\mathbf{0 . 8 0 7 0 0}$ \\
T-4 & 28.12420 & 100.15212 & 5.21739 & 0.85886 & 22.49632 & 365.97249 & 10.88982 & 0.63714 \\
T-5 & 27.19469 & 124.05425 & 7.54450 & 0.70633 & 26.86688 & 133.78033 & 7.73621 & 0.70899 \\
T-6 & 26.95957 & 130.95526 & 7.79282 & 0.69942 & 26.50296 & 145.47357 & 8.05051 & 0.69856
\end{tabular}


Pradeep K. Gupta, Shyam Lal and Farooq Husain/Journal of Engineering Science and Technology Review 13 (5) (2020) 20 - 32

\begin{tabular}{c|c|c|c|c|c|c|c|c} 
T-7 & 25.51974 & 182.43404 & 2.61490 & 0.98123 & 23.63541 & 281.53967 & 7.93047 & 0.82920 \\
T-8 & 18.44191 & 930.87064 & 7.65002 & 0.90865 & 18.58495 & 900.71114 & 8.53813 & 0.86397 \\
T-9 & 26.14453 & 157.98906 & 8.28071 & 0.79278 & 23.14360 & 315.29768 & 10.67578 & 0.73581 \\
T-10 & 32.39232 & 37.48425 & 3.77489 & 0.92303 & 29.15688 & 78.95730 & 5.15594 & 0.88176 \\
\hline
\end{tabular}

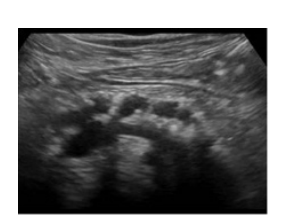

Real Image

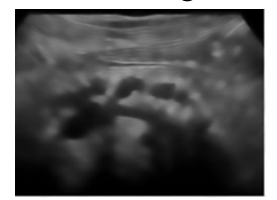

T-5

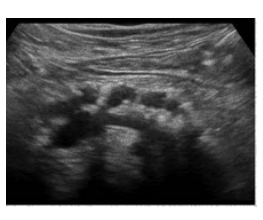

Noisy Image

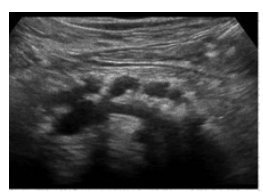

T-6

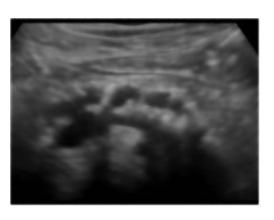

T-1

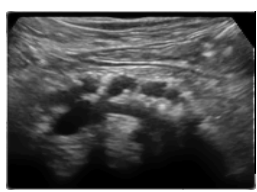

T-7

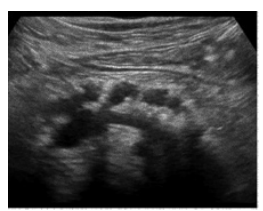

T-2

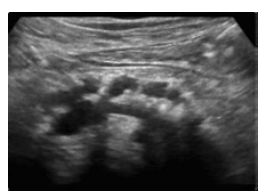

T-8

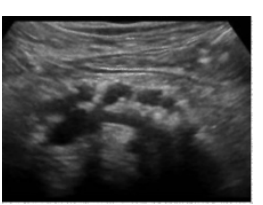

T-3

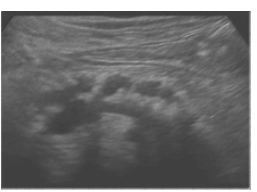

T-9

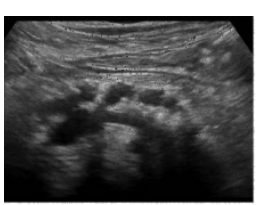

T-4

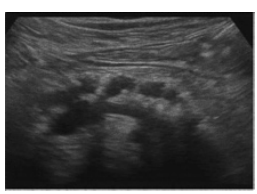

$\mathrm{T}-10$

Fig. 9. Comparative visual performance of pancreas image at noise variance for the value of $\sigma=0.10$

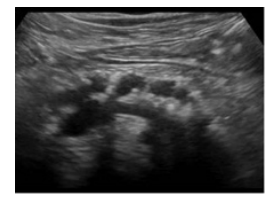

Real Image

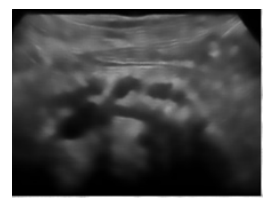

T-5

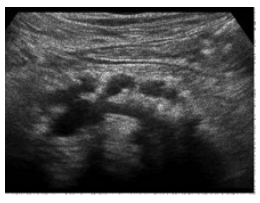

Noisy Image

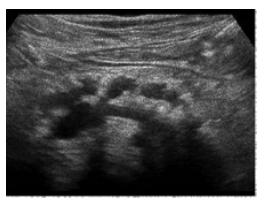

T-6

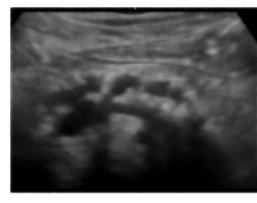

$\mathrm{T}-1$

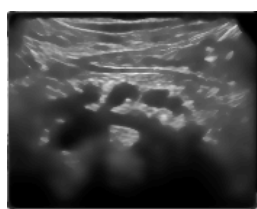

T-7

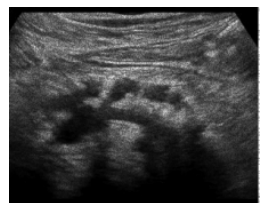

T-2

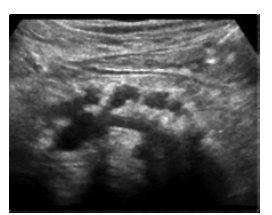

T-8

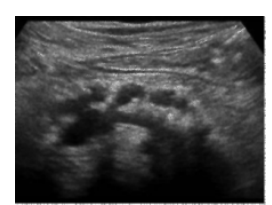

T-3

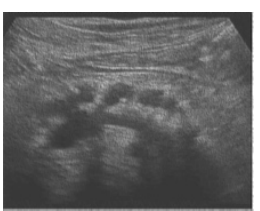

T-9

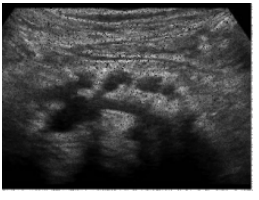

T-4

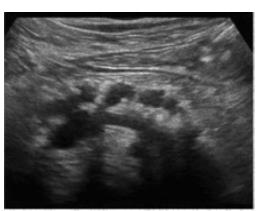

$\mathrm{T}-10$

Fig. 10. Comparative visual performance of pancreas image at noise variance for the value of $\sigma=0.20$

Table 5. Comparative Quality Measures values for parathyroid gland image

\begin{tabular}{c|c|c|c|c|c|c|c|c}
\hline & \multicolumn{4}{|c|}{$\boldsymbol{\sigma}=\mathbf{0 . 1 0}$} & \multicolumn{3}{c}{$\boldsymbol{\sigma}=\mathbf{0 . 2 0}$} \\
\hline Filter & PSNR & MSE & MAE & SSIM & PSNR & MSE & MAE & SSIM \\
\hline T-1 & 22.87459 & 335.44530 & 8.99179 & 0.74525 & 22.69176 & 349.86787 & 8.84239 & 0.76235 \\
T-2 & 11.32876 & 4788.55470 & 52.80384 & 0.04285 & 11.32793 & 4789.47221 & 52.80383 & 0.04286 \\
T-3 & 32.08095 & 40.27031 & 4.02338 & 0.91152 & 29.24273 & 77.41184 & 5.16849 & 0.87849 \\
T-4 & 29.05217 & 80.88418 & 4.61425 & 0.89010 & 23.28434 & 305.24364 & 9.62673 & 0.69888 \\
T-5 & 27.05720 & 128.04439 & 7.66175 & 0.70428 & 26.61564 & 141.74782 & 7.86830 & 0.70587 \\
T-6 & 27.05288 & 128.17187 & 7.65316 & 0.71092 & 26.60066 & 142.23774 & 7.88946 & 0.71150 \\
T-7 & 26.51541 & 145.05724 & 2.38254 & 0.97219 & 26.18591 & 156.49082 & 2.77746 & 0.96496 \\
T-8 & 19.18250 & 784.92826 & 7.52026 & 0.89824 & 19.05468 & 808.37445 & 8.68883 & 0.85987 \\
T-9 & 27.93951 & 104.50306 & 5.62315 & 0.90085 & 23.42080 & 295.80125 & 9.83982 & 0.75018 \\
\hline T-10 & $\mathbf{3 3 . 5 9 4 2 2}$ & $\mathbf{2 8 . 4 2 2 2 5}$ & $\mathbf{3 . 5 1 6 6 3}$ & $\mathbf{0 . 9 3 8 3 9}$ & $\mathbf{2 8 . 7 2 3 4 6}$ & $\mathbf{8 7 . 2 4 3 7 5}$ & $\mathbf{6 . 3 6 1 1 5}$ & $\mathbf{0 . 8 3 6 9 3}$ \\
\hline
\end{tabular}

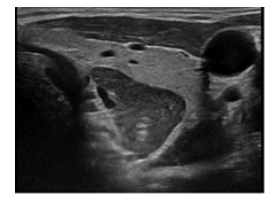

Real Image

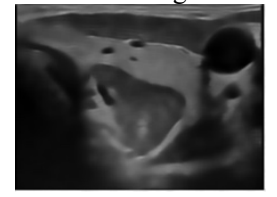

T-5

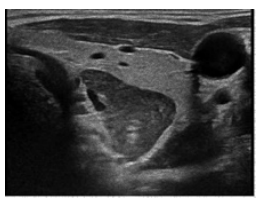

Noisy Image

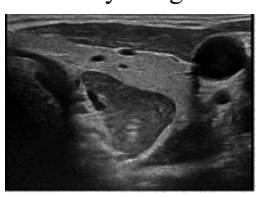

T-6

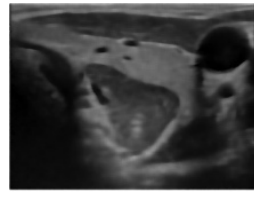

$\mathrm{T}-1$

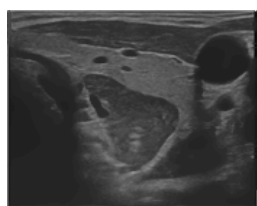

T-7

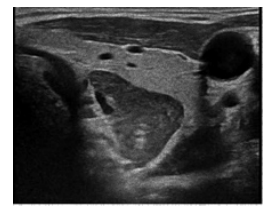

$\mathrm{T}-2$

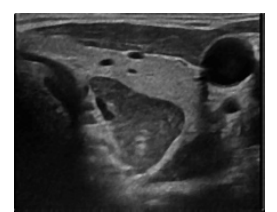

$\mathrm{T}-8$

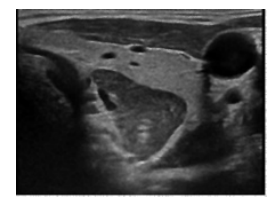

$\mathrm{T}-3$

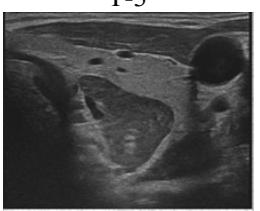

T-9

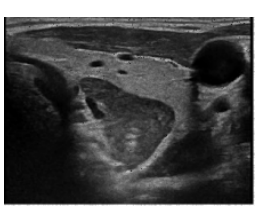

$\mathrm{T}-4$

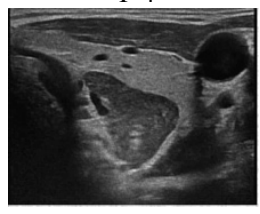

$\mathrm{T}-10$

Fig. 11. Comparative visual performance of parathyroid gland image at noise variance $\sigma=0.10$ 


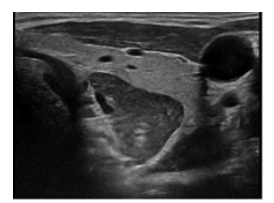

Real Image

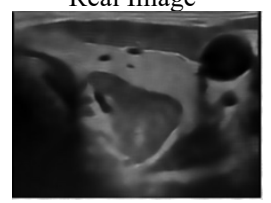

$\mathrm{T}-5$

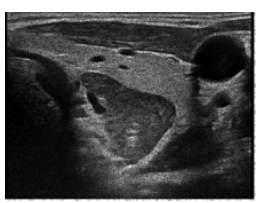

Noisy Image

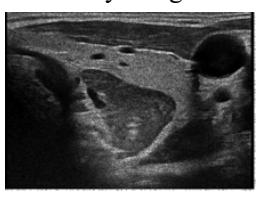

T-6

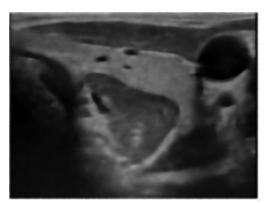

$\mathrm{T}-1$

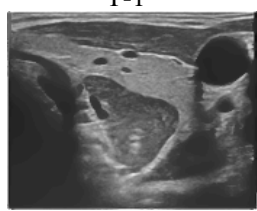

$\mathrm{T}-7$

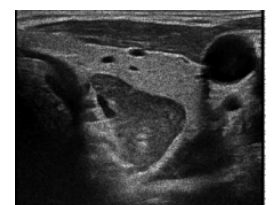

T-2

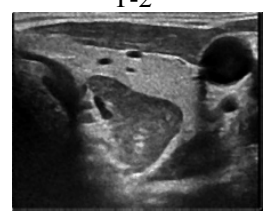

T-8

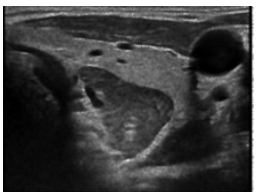

T-3

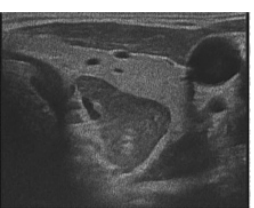

T-9

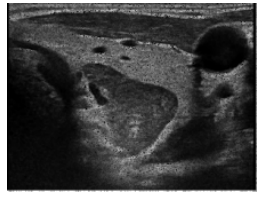

$\mathrm{T}-4$

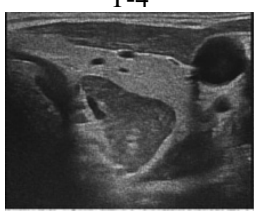

T-10

Fig. 12. Comparative visual performance of parathyroid gland image at noise variance $\sigma=0.20$

Table 6. Comparative Quality Measures values for scrotal trauma image

\begin{tabular}{c|c|c|c|c|c|c|c|c}
\hline & \multicolumn{4}{|c|}{$\boldsymbol{\sigma}=\mathbf{0 . 1 0}$} & \multicolumn{4}{c}{$\boldsymbol{\sigma}=\mathbf{0 . 2 0}$} \\
\hline Filter & PSNR & MSE & MAE & SSIM & PSNR & MSE & MAE & SSIM \\
\hline T-1 & 23.56757 & 285.97212 & 8.89221 & 0.71653 & 23.50712 & 289.98006 & 8.37467 & 0.75757 \\
T-2 & 9.16201 & 7886.41917 & 73.75492 & 0.01518 & 9.16154 & 7887.27095 & 73.75491 & 0.01521 \\
T-3 & 30.84990 & 53.46754 & 5.21339 & 0.85409 & 26.50365 & 145.45065 & 8.34377 & 0.74283 \\
T-4 & 27.68515 & 110.80647 & 6.36455 & 0.79975 & 20.70806 & 552.42636 & 13.74739 & 0.54582 \\
T-5 & 27.69313 & 7.70625 & 110.60317 & 0.67021 & 27.21151 & 123.57453 & 8.00156 & 0.67268 \\
T-6 & 26.94320 & 131.44978 & 8.28371 & 0.65850 & 26.48751 & 145.99206 & 8.53535 & 0.65736 \\
T-7 & 24.95923 & 207.56672 & 6.15672 & 0.85519 & 22.68391 & 350.50096 & 10.44921 & 0.68150 \\
T-8 & 19.17972 & 785.43192 & 7.64295 & 0.89607 & 19.65715 & 703.66427 & 8.61831 & 0.83262 \\
T-9 & 23.41212 & 296.39316 & 10.94818 & 0.72201 & 25.70946 & 174.63611 & 7.69102 & 0.79123 \\
\hline T-10 & $\mathbf{3 3 . 0 8 0 6 0}$ & $\mathbf{3 1 . 9 9 0 4 4}$ & $\mathbf{3 . 8 2 5 9 1}$ & $\mathbf{0 . 9 1 5 9 6}$ & $\mathbf{2 8 . 1 6 7 7 0}$ & $\mathbf{9 9 . 1 5 4 0 0}$ & $\mathbf{6 . 1 5 8 2 4}$ & $\mathbf{0 . 8 2 7 7 7}$ \\
\hline
\end{tabular}

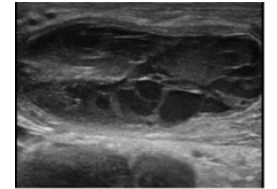

Real Image

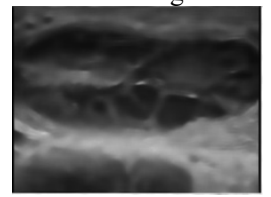

T-5

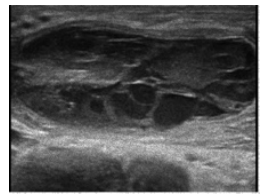

Noisy Image

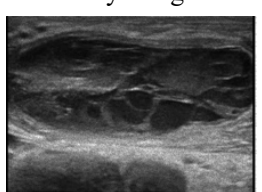

T-6

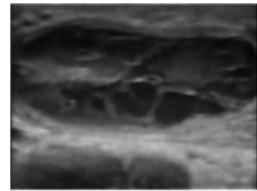

$\mathrm{T}-1$

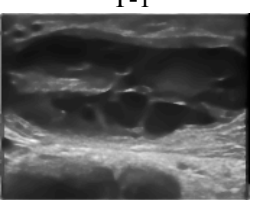

T-7

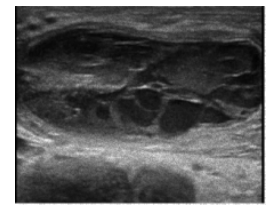

$\mathrm{T}-2$

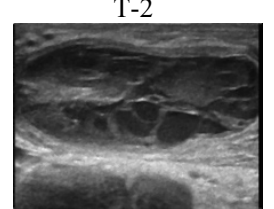

T-8

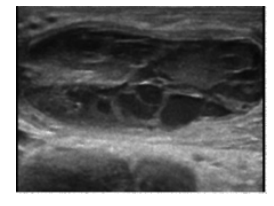

$\mathrm{T}-3$

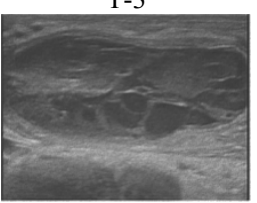

T-9
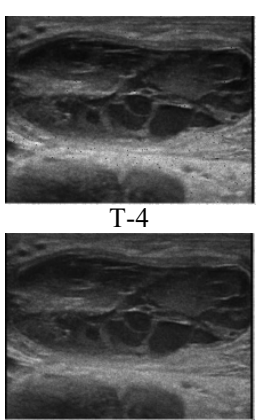

$\mathrm{T}-10$

Fig. 13. Comparative visual performance of scrotal trauma image at noise variance $\sigma=0.10$

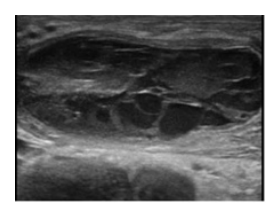

Real Image

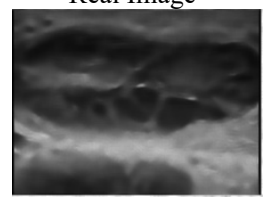

T-5

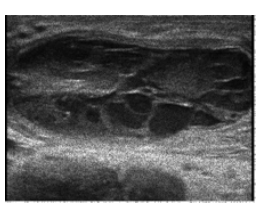

Noisy Image

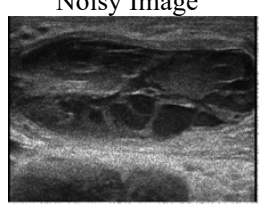

T-6

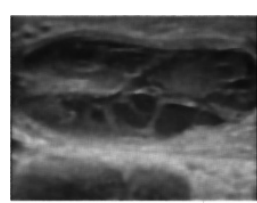

T-1

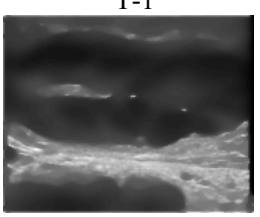

T-7

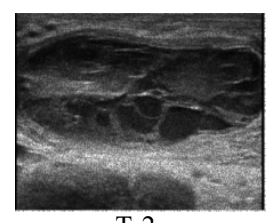

T-2

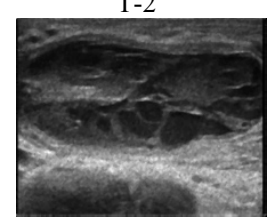

T-8

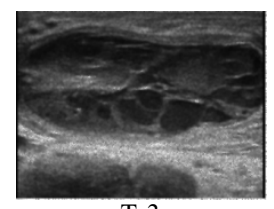

T-3

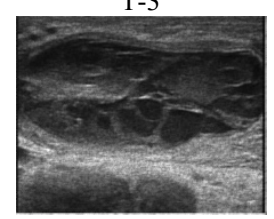

T-9

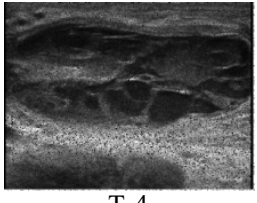

T-4

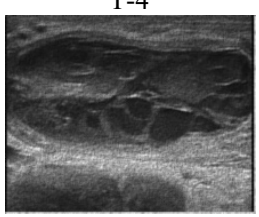

T-10

Fig. 14. Comparative visual performance of scrotal trauma image at noise variance $\sigma=0.20$

Table 7. Comparative Quality Measures values for Thoracic wall image

\begin{tabular}{c|c|c|c|c|c|c|c|c}
\hline & \multicolumn{4}{|c|}{$\boldsymbol{\sigma}=\mathbf{0 . 1 0}$} & \multicolumn{4}{c}{$\boldsymbol{\sigma}=\mathbf{0 . 2 0}$} \\
\hline Filter & PSNR & MSE & MAE & SSIM & PSNR & MSE & MAE & SSIM \\
\hline T-1 & 23.65584 & 280.21810 & 8.69644 & 0.76072 & 23.46404 & 292.87068 & 8.59664 & 0.77462 \\
T-2 & 11.30483 & 4815.01368 & 52.68119 & 0.02722 & 11.30369 & 4816.27052 & 52.68142 & 0.02725 \\
T-3 & 31.98635 & 41.15716 & 3.97073 & 0.92283 & 28.53348 & 91.14485 & 6.19464 & 0.85127 \\
T-4 & 28.07672 & 101.25302 & 4.70073 & 0.89397 & 22.67090 & 351.55287 & 9.79836 & 0.70747 \\
T-5 & 26.60269 & 142.17098 & 7.78141 & 0.71957 & 26.04464 & 161.66514 & 8.07021 & 0.72072
\end{tabular}


Pradeep K. Gupta, Shyam Lal and Farooq Husain/Journal of Engineering Science and Technology Review 13 (5) (2020) 20 - 32

\begin{tabular}{c|c|c|c|c|c|c|c|c} 
T-6 & 26.63330 & 141.17246 & 7.80292 & 0.73809 & 26.04971 & 161.47634 & 8.09929 & 0.73691 \\
T-7 & 20.50504 & 578.86399 & 4.61377 & 0.92140 & 22.40133 & 374.06555 & 4.98652 & 0.89927 \\
T-8 & 20.19739 & 621.35753 & 6.96510 & 0.91068 & 20.10861 & 634.18948 & 7.70413 & 0.88084 \\
T-9 & 28.75799 & 86.55276 & 5.15700 & 0.92437 & 22.59041 & 358.12874 & 12.42809 & 0.61407 \\
\hline T-10 & $\mathbf{3 3 . 0 4 1 4 4}$ & $\mathbf{3 2 . 2 8 0 2 4}$ & $\mathbf{3 . 8 0 4 5 9}$ & $\mathbf{0 . 9 3 7 3 7}$ & $\mathbf{2 8 . 5 6 0 5 6}$ & $\mathbf{9 0 . 5 7 8 3 7}$ & $\mathbf{5 . 4 1 0 4 9}$ & $\mathbf{0 . 8 8 7 4 2}$ \\
\hline
\end{tabular}
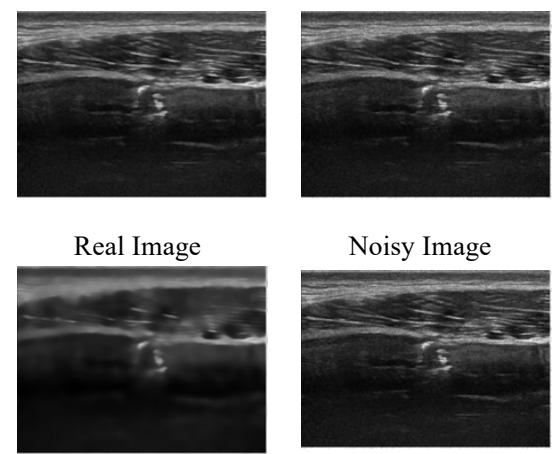

T-5

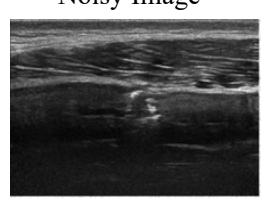

T-6

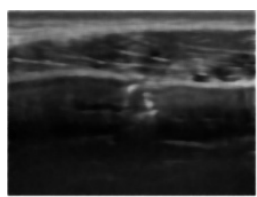

$\mathrm{T}-1$

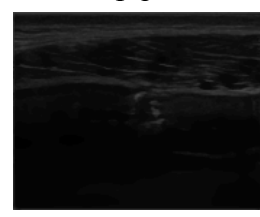

T-7

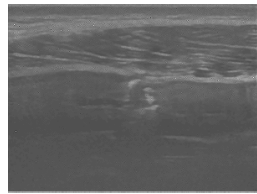

$\mathrm{T}-2$

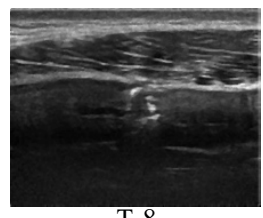

T-8

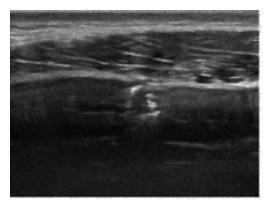

$\mathrm{T}-3$

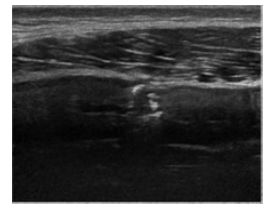

T-9
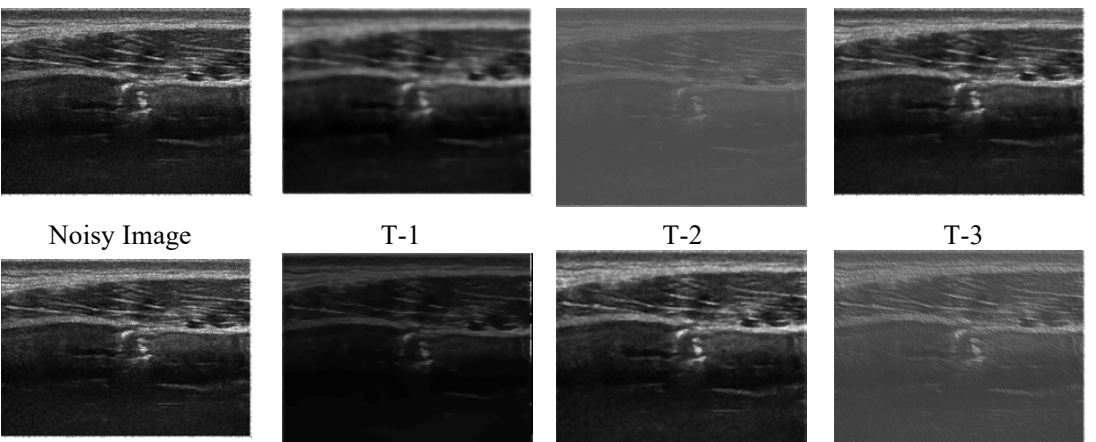

T-6

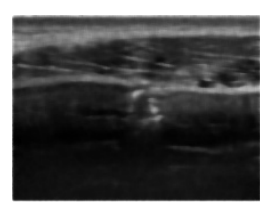

$\mathrm{T}-1$

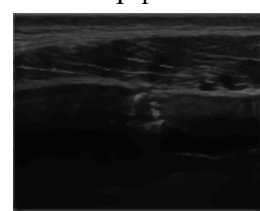

T-7

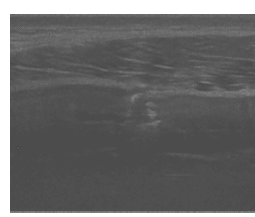

$\mathrm{T}-2$

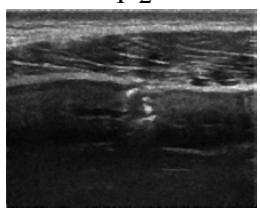

$\mathrm{T}-8$
T-3

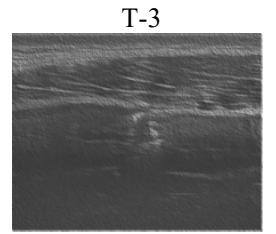

T-9

Fig. 16. Comparative visual performance of Thoracic wall image at noise variance $\sigma=0.20$

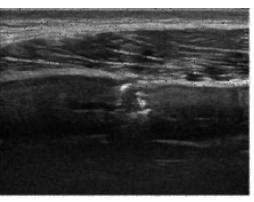

$\mathrm{T}-4$

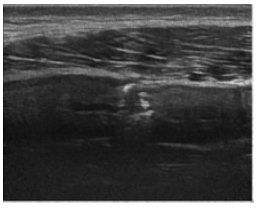

T-10

Table 8. Comparative Quality Measures values for uterus image

\begin{tabular}{c|c|c|c|c|c|c|c|c}
\hline & \multicolumn{4}{|c}{$\boldsymbol{\sigma}=\mathbf{0 . 1 0}$} & \multicolumn{4}{c}{$\boldsymbol{\sigma}=\mathbf{0 . 2 0}$} \\
\hline Filter & PSNR & MSE & MAE & SSIM & PSNR & MSE & MAE & SSIM \\
\hline T-1 & 22.90182 & 333.34853 & 7.82545 & 0.78269 & 22.72182 & 347.45502 & 7.67114 & 0.79531 \\
T-2 & 11.32440 & 4793.36278 & 45.34843 & 0.27540 & 11.32332 & 4794.55866 & 45.34840 & 0.27546 \\
T-3 & 30.86096 & 53.33153 & 4.32112 & 0.89798 & 28.47911 & 92.29306 & 5.91606 & 0.81796 \\
T-4 & 27.90631 & 105.30501 & 4.15858 & 0.89640 & 22.55270 & 361.25236 & 8.58462 & 0.74687 \\
T-5 & 27.85990 & 106.43649 & 6.12200 & 0.75613 & 27.34075 & 119.95133 & 6.37750 & 0.75732 \\
T-6 & 27.34979 & 119.70189 & 6.48463 & 0.74508 & 26.80808 & 135.60385 & 6.77318 & 0.74503 \\
T-7 & 27.29878 & 121.11624 & 1.11894 & 0.99541 & 27.14924 & 125.35939 & 1.22151 & 0.99408 \\
T-8 & 18.75033 & 867.05670 & 7.42265 & 0.91253 & 18.62626 & 892.18437 & 7.89001 & 0.88112 \\
T-9 & 23.84467 & 268.29553 & 9.49959 & 0.79011 & 21.50988 & 459.29521 & 12.08215 & 0.68693 \\
\hline T-10 & $\mathbf{3 2 . 1 6 5 1 2}$ & $\mathbf{3 9 . 4 9 7 3 8}$ & $\mathbf{3 . 5 1 5 0 4}$ & $\mathbf{0 . 9 2 4 1 3}$ & $\mathbf{2 8 . 8 0 7 6 1}$ & $\mathbf{8 5 . 5 6 9 6 0}$ & $\mathbf{4 . 7 5 5 7 9}$ & $\mathbf{0 . 8 9 1 4 0}$ \\
\hline
\end{tabular}

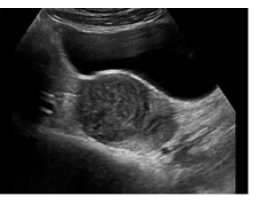

Real Image

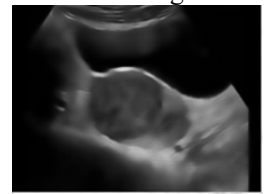

T-5

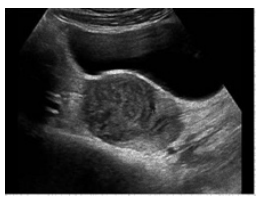

Noisy Image

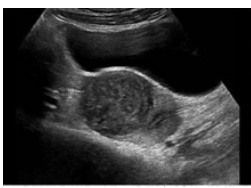

T-6

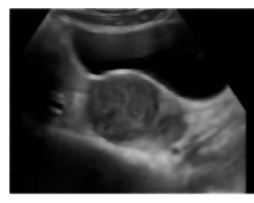

$\mathrm{T}-1$

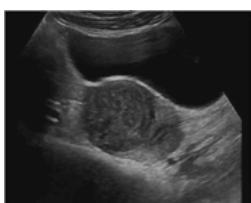

$\mathrm{T}-7$

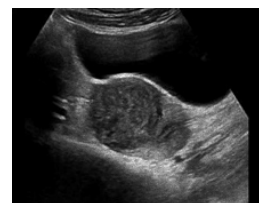

$\mathrm{T}-2$

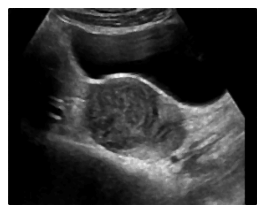

$\mathrm{T}-8$

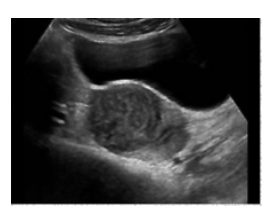

$\mathrm{T}-3$

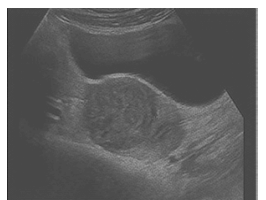

T-9

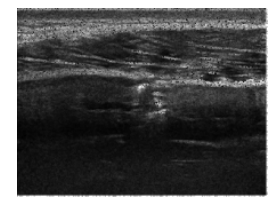

$\mathrm{T}-4$

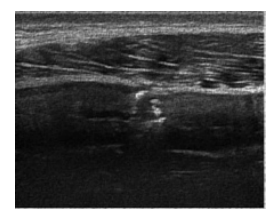

T-10

Fig. 17. Comparative visual performance of uterus image at noise variance $\sigma=0.10$ 


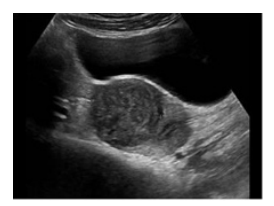

Real Image

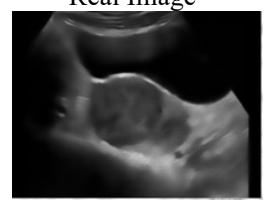

T-5

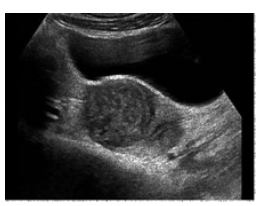

Noisy Image

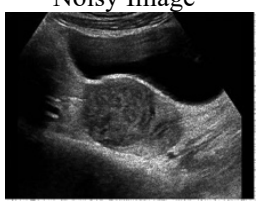

T-6

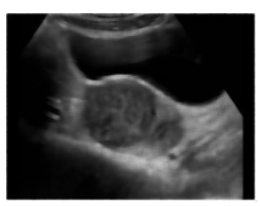

$\mathrm{T}-1$

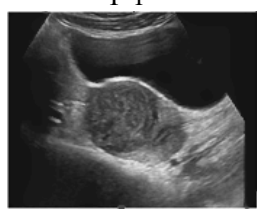

$\mathrm{T}-7$

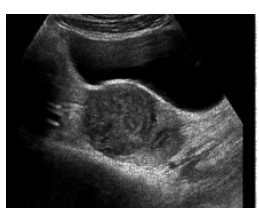

T-2

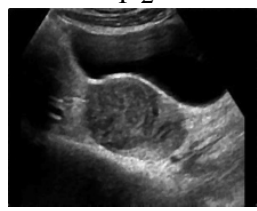

$\mathrm{T}-8$

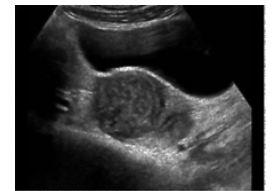

$\mathrm{T}-3$

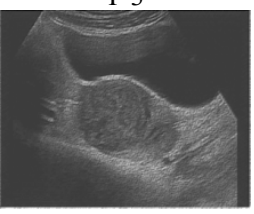

T-9

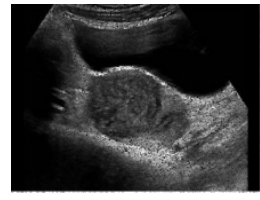

$\mathrm{T}-4$

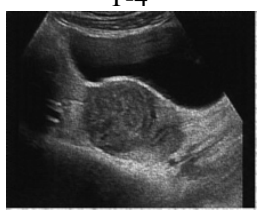

$\mathrm{T}-10$

Fig. 18 Comparative visual performance of uterus image at noise variance $\sigma=0.20$

filtering approach show that the output images have high quality along with fine detail in comparison to other benchmark despeckling filters. The output results obtained by the proposed filter are not much blurred even when we increase the noise level. The visual results obtained by the proposed filtering approach are smoother, less noisy for different noise variance levels in comparison to other algorithms. The better visual results of the proposed filtering framework are also supported by the different quality measures. Table 1 to Table 8 are shows the comparative quality measures for the images of adrenal gland, appendicitis, bladder, pancreas, parathyroid gland, scrotal gland, thoracic wall, and uterus, respectively. The lowest value of MSE and MAE for the proposed filtering approach proves the worthiness of the proposed filtering framework.

Out of different quality measures, the PSNR, SSIM values are significant criteria to analyze the comparative quality and similarity between the noisy and denoise images. The highest value of PSNR and SSIM also supports the visual results of the proposed system. In addition to this, noise suppression capability and better edge preservation capability of the proposed filtering approach in comparison to other methods are also justified. Therefore, we can say that the proposed filtering approach satisfies the requirement of an excellent despeckling approach, so that the proposed filtering approach may play a vital role in the field of medical image enhancement and help in the proper diagnosis of patients. Therefore, the results of the proposed filtering approach indicated that the proposed filtering framework is better than benchmark despeckling methods. The proposed algorithm improves the quality of the image, along with restoring the relevant edge information of the image.

\subsection{Effect of FNLM filtering}

In this proposed framework, we use the FNLM filter as a postprocessing filter. Table 9 shows the PSNR, MSE, MAE, and SSIM value of the proposed approach without FNLM ,from the values; it is clear that the FNLM filter plays an essential role in improving the output results of the proposed framework. Fig.19 and Fig.20 shows the visual impacts of FNLM filter in the proposed framework for different image of adrenal gland, appendicitis, bladder at different noise variance.

Table 9. Performance Analysis of Proposed filter with or without FNLM filter

\begin{tabular}{|c|c|c|c|c|c|c|c|c|}
\hline \multirow{2}{*}{ Image } & \multicolumn{4}{|c|}{$\sigma=0.10$} & \multicolumn{4}{|c|}{$\sigma=0.20$} \\
\hline & PSNR & MSE & MAE & SSIM & PSNR & MSE & MAE & SSIM \\
\hline $\begin{array}{c}\text { Adrenal Gland } \\
\text { (without FNLM) }\end{array}$ & 30.24217 & 61.49834 & 5.77514 & 0.86746 & 25.89056 & 167.50366 & 8.68197 & 0.75030 \\
\hline $\begin{array}{l}\text { Adrenal Gland } \\
\text { (with FNLM) }\end{array}$ & 31.26479 & 48.59608 & 4.89910 & 0.90558 & 28.60008 & 89.75775 & 6.76960 & 0.75976 \\
\hline $\begin{array}{c}\text { Appendicitis } \\
\text { (Without FNLM) }\end{array}$ & 30.75858 & 54.60368 & 4.92239 & 0.90734 & 27.21912 & 123.35823 & 7.23972 & 0.80487 \\
\hline $\begin{array}{l}\text { Appendicitis } \\
\text { (With FNLM) }\end{array}$ & 31.88799 & 42.09996 & 4.49206 & 0.92109 & 29.65441 & 70.41077 & 5.85625 & 0.81860 \\
\hline $\begin{array}{c}\text { Bladder } \\
\text { (Without FNLM) }\end{array}$ & 30.66581 & 44.30979 & 3.93530 & 0.89470 & 26.97366 & 130.53134 & 6.66783 & 0.77130 \\
\hline $\begin{array}{c}\text { Bladder } \\
\text { (With FNLM) }\end{array}$ & 30.93989 & 52.37106 & 4.30781 & 0.87160 & 29.94910 & 65.79166 & 4.74363 & 0.81392 \\
\hline
\end{tabular}

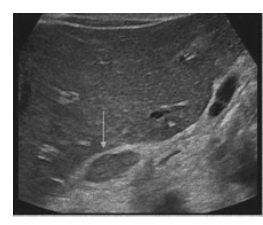

Adrenal Gland without FNLM

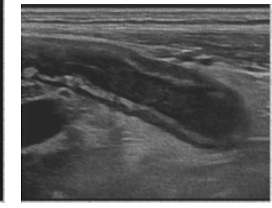

Appendicitis without FNLM

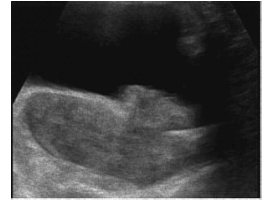

bladder without FNLM

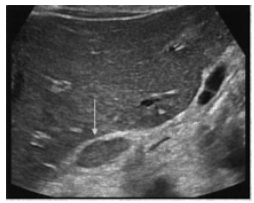

Adrenal Gland with FNLM

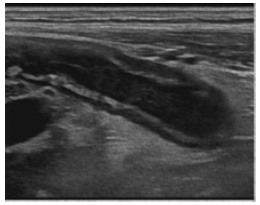

Appendicitis with FNLM

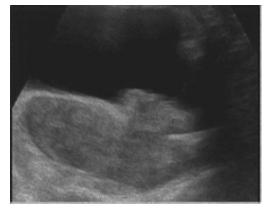

bladder with FNLM

Fig. 19. Effect of FNLM filtering on the images for the value of $\sigma=0.10$ 


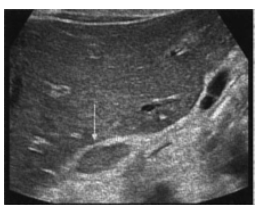

Adrenal Gland

without FNLM

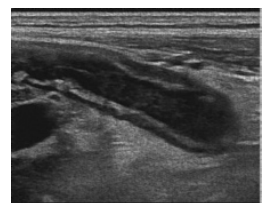

Appendicitis without FNLM

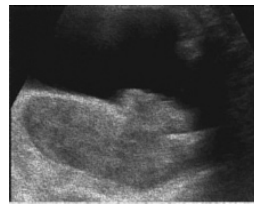

bladder without FNLM

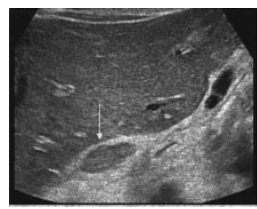

Adrenal Gland with FNLM

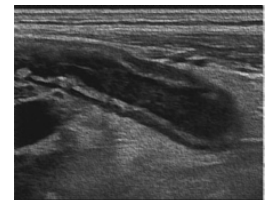

Appendicitis with FNLM

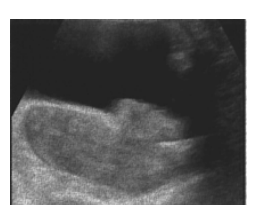

bladder with FNLM

Fig. 20. Effect of FNLM filtering on the images for the value of $\sigma=0.20$

\subsection{Time Consuming Analysis}

The time-consuming analysis is also performing to give a fair comparison of the proposed framework with other benchmark despeckling algorithms compared. Table 10 shows the time-consuming analysis of the proposed framework with other compared filters. Table indicate that the proposed framework consume $6.49 \mathrm{sec}$ in comparison to filters T-1,T-2,T-3,T-4,T-5,T-6,T-7,T-8 and T-9 which are consume 2.90,14.22,1.27,28.08,7.62,8.14,7.60,13.26, and 12.54 respectively. Fig.21 represent the graphical comparison of time analysis of proposed framework.From the results, it is clear that the proposed framework consumes more time in comparison to T-1 and T-3 filters but takes less time in comparison to all other remaining filters. So, we conclude that the proposed framework is also a speedy framework for despeckling of real ultrasound images.

Table 10. Time Analysis of Proposed Framework

\begin{tabular}{l|c|c|c|c|c|c|c|c|c|c}
\hline Filter & T-1 & T-2 & T-3 & T-4 & T-5 & T-6 & T-7 & T-8 & T-9 & T-10 \\
\hline Time(sec) & 2.90 & 14.22 & 1.27 & 28.08 & 7.62 & 8.14 & 7.60 & 13.26 & 12.54 & 6.49 \\
\hline
\end{tabular}

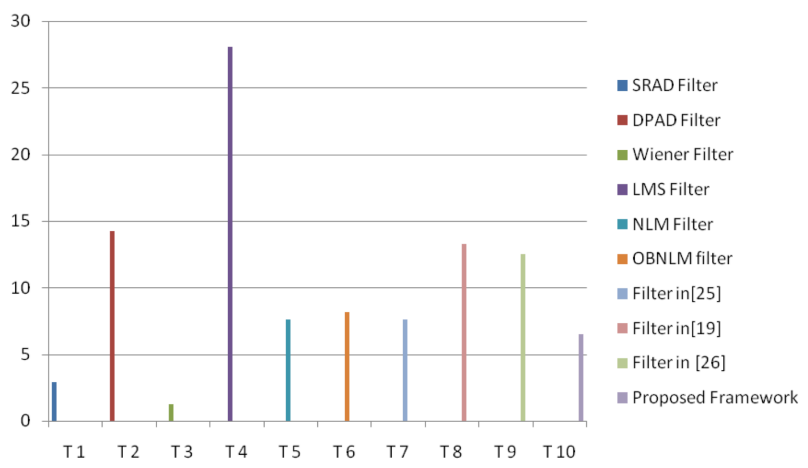

Fig. 21. Time analysis of proposed framework in comparison to all other compared filters

\section{Conclusion}

To improve the diagnosis finding of disease from real ultrasound images, it is pre-requisites to enhance the images. Thus, in this research work, we proposed a robust despeckling framework that mitigates the effect of speckle noise and gives a better-enhanced output image. In this approach, we used $\mathrm{ABC}$ optimization algorithm to find out the optimum value of the filter coefficient. To prove the worthiness of proposed framework, the different quality matrices value were calculated by performing the experiments on different real ultrasound images of adrenal gland, appendicitis, bladder, pancreas, parathyroid gland, scrotal gland, thoracic wall, and uterus. Noisy images were obtained by noise intensity (i.e., $0.10,0.20)$ in the original ultrasound image. The quantitative comparative performance analysis of the proposed framework with other benchmark despeckling algorithms were elaborated by calculating the PSNR, MSE, MAE, and SSIM values. To make a fair comparison between proposed filtering approach and other benchmark deseckling algorithms, all require parameters were fixed as suggested by the respective authors in the literature. The numerical and visual results of the proposed framework were better in comparison to other benchmark despeckling algorithms. The proposed framework successfully removes noise while preserving the important information of images. The main advantage of the proposed framework is, its low computational complexity and a fixed number of iterations that make it a better choice for the reduction of speckle noise. The limiting factor of our proposed framework is its objective function. We consider the MSE only an objective function for our proposed approach. Instead of MSE as an objective function, other quality measures may be used as an objective function to improve the results further. We have conducted an experiment only on real ultrasound images; in the future other images may be tested for further study.

This is an Open Access article distributed under the terms of the Creative Commons Attribution License

\section{References}

[1]. T. L. Szabo, Diagnostic Ultrasound Imaging: Inside Out (Biomedical Engineering). Amsterdam, The Netherlands: Elsevier (2004)

[2]. J. M. Thijssen, B. J. Oosterveld, Texture in tissue echograms, Speckle or information?,J.Ultrasound Med.,vol. 9, no. 4, pp. 215229, (1990).

[3]. T. Loupas, W. Mc Dicken, P. Allan, An adaptive weighted median filter for speckle suppression in medical ultrasound images, IEEE Transactions on Circuits and Systems, vol. 36, no. 1, pp.129-135, (1989).
[4]. D. Hillery, R.T. Chin, Iterative Wiener filters for images restoration, IEEE Transaction on Signal Processing, vol.39, pp.1901-1904, (1991).

[5]. J. W. Goodman, Some fundamental properties of speckle, J. Opt. Soc. Amer., vol. 66, no. 11, pp. 1145-1150,(1976).

[6]. J. S. Lee, Digital Image Enhancement and noise filtering by use of local statistics, IEEE Trans Pattern Anal mach Intell., vol.2, no. 2, pp.165-68, (1980).

[7]. V. S. Frost, J. A. Stiles, K. S. Shanmugan and J. Holtzman, A Model for Radar Images and Its Application to adaptive Digital Filtering 
of Multiplicative Noise, IEEE trans. Pattern anal Mach intell, vol.4, no.2, pp.157-66, (1982).

[8]. D. Kaun, A. Sawchuck, T. Strand, P. Chaved, Adaptive noise smoothing filter for images with signal -dependent noise, IEEE Trans Pattern Anal mach Intell, vol. 7, no. 2, pp.165-77, (1985).

[9]. A. Lopes, E. Nezry, R.Touzi, and H. laur, Maximum a posteriori speckle filtering and first order texture models in SAR images, In Proc. $10^{\text {th }}$ Int. Geosci. Remote Sens. Symp., , pp.2409-2412, May (1990)

[10]. Y. Yu and S. T. Acton, Speckle reducing anisotropic diffusion, IEEE Trans. Image Process., vol. 11, no. 11, pp. 1260-1270, Nov. (2002).

[11]. S. Aja-Fernández and C. Alberola-López, On the estimation of the coefficient of variation for anisotropic diffusion speckle filtering, IEEE Trans. Image Process., vol. 15, no. 9, pp. 2694-2701, Sep. (2006).

[12]. K. Krissian, C.-F. Westin, R. Kikinis, and K. G. Vosburgh, Oriented speckle reducing anisotropic diffusion, IEEE Trans. Image Process.,vol. 16, no. 5, pp. 1412-1424, ( 2007).

[13]. G. Vegas-Sanchez-Ferrero, S. Aja-Fernandez, M. Martin-Fernandez, A. F. Frangi, and C. Palencia, Probabilistic-driven oriented speckle reducing anisotropic diffusion with application to cardiac ultrasonic images, in Proc. 13th Int. Conf. Med. Image Comput. Comput. Assist. Intervent., pp. 518-525, (2010).

[14]. P. Coupe, P. Hellier, C. Kervrann, and C. Barillot, Nonlocal means based speckle filtering for ultrasound images, IEEE Trans. ImageProcess., vol. 18, no. 10, pp. 2221-2229, (2009).

[15]. V. Soni, A. K. Bhandari, A. Kumar, G. K. Singh, Improved subband adaptive thresholding function for denoising of satellite image based on evolutionary algorithms, IET Signal Processing, vol. 7, no. 8, pp.720-730, (2013).

[16]. JL de Paiva, CF Toledo, H Pedrini, An Approach Based on Hybrid Genetic Algorithm Applied To Image Denoising Problem, Elsevier journal of Applied Soft Computing, vol. 46, pp.778-91, (2016).
[17]. M Malik, F Ahsan, S Mohsin, Adaptive image denoising using cuckoo algorithm, Springer journal of Soft Computing, vol. 20, pp. 925-38, (2016).

[18]. K. Boudjelaba, D. Chikouche, and F. Ros, Evolutionary techniques for the synthesis of 2-D FIR filter, IEEE Statistical Signal Processing Workshop (SSP), pp, 601-604, (2011).

[19]. F. Latifoglu A Novel Approach to Speckle Noise Filtering Based On Artificial Bee Colony Algorithm: Ultrasound Image Applications, Computer Methods and Progress In Biomedicine, vol. 111, no.3, pp. 561-69, (2013).

[20]. D. Karaboga, An Idea based on honey bee swarm for numerical optimization, Technical report-TR06, Erciyes University, Engineering Faculty, Computer Engineering Department, (2005).

[21]. A Buades, B Coll , JM Morel, A Review Of Image Denoising Algorithms With A New one, Multiscale Model Simul, vol.4, no. 2, pp.490-530, (2005).

[22]. D. Karaboga, Artificial Bee Colony Algorithm, Scholarpedia: www.scholarpedia.org/articleArtificial bee colony algorithm, (2010).

[23]. M.M. Hadhoud, D.W. Thomas, The two-dimensional adaptive LMS algorithm, IEEE Trans. Circuits Syst., vol.35, no.5, pp.485-494, (1988).

[24]. AM Atto, D Pastor, G Mercier, Smooth Adaptation by Sigmoid Shrinkage, EURASIP Journal on Image and Video Process, (2009).

[25]. G Ramos-Llorden, G Vegas-Sánchez-Ferrero, M Martin-Fernandez et al., Anisotropic Diffusion filter with Memory Based on speckle Statistics for Ultrasound Images, IEEE Trans on Image Processing, vol. 24, no. 1, pp.345-58, (2015).

[26]. S. Kockanata, N. Karaboga, A novel 2D-ABC adaptive filter algoritm: A comparative study, Elsevier journal of Digital Signal Processing, vol.40, pp.140-53, (2015).

[27]. W. Zhou, B.A. Conrad, S.H. Rahim, Image Quality assessment: from error visibility to structural similarity, IEEE Trans on Image Process, vol.13 no. 4, pp.600-12, (2004). 